\title{
Determination of the X-Ray Elastic Constants of the Ti-6AI-4V Processed by Powder Bed-Laser Beam Melting
}

\author{
Nathan Dumontet ${ }^{1, a,{ }^{*}}$, Guillaume Geandier ${ }^{2, b}$, Florian Galliano ${ }^{3, c}$, \\ Bernard Viguier ${ }^{1, d}$ and Benoit Malard ${ }^{1, e}$ \\ ${ }^{1}$ CIRIMAT, Université de Toulouse, CNRS, INP- ENSIACET 4 allée Emile Monso - BP44362, \\ 31030 Toulouse Cedex 4 - France \\ ${ }^{2}$ Institut Jean Lamour, UMR 7198 CNRS - Université de Lorraine, Campus ARTEM 2 allée \\ André Guinier BP 5084054011 Nancy Cedex France \\ ${ }^{3}$ MBDA France, Site de Bourges Aeroport, Route d'Issoudun, 18030 Bourges Cedex' France \\ anathan.dumontet@ensiacet.fr, bguillaume.geandier@univ-lorraine.fr, \\ cflorian.galliano@mbda-systems.com, ${ }^{\mathrm{d}}$ bernard.viguier@ensiacet.fr, ${ }^{\mathrm{e}}$ benoit.malard@ensiacet.fr
}

Keywords: X-Ray Elastic Constants, Laser Beam Melting, Martensitic Phase, Ti64

\begin{abstract}
The determination of residual stresses (RS) through X-Ray Diffraction (XRD) necessitates the knowledge of the X-Ray Elastic Constants (XEC) $S_{1}$ and $1 / 2 S_{2}$ for the specific phase in presence. In the case of Ti-6Al-4V additive manufacturing (AM) elaboration method such as Powder Bed Laser Beam Melting (PB-LBM) results in a microstructure fully constituted $\alpha^{\prime}$ martensitic phase. The properties of this $\alpha^{\prime}$ martensite are largely unknown. The purpose of the present paper is to determine the XEC of the $\alpha$ ' martensitic phase. This is performed using an original methodology involving 2D X-Ray detection. The results shows significant differences with XEC values available for the $\alpha$ phase from Titanium.
\end{abstract}

\section{Introduction}

AM is a process developed to build parts with less building steps than conventional manufacturing routes. There exists a lot of different processes for AM, among them the LBM consists in melting a thin metallic layer of powder using a localized laser beam and building layer by layer a part in three dimensions. Because of the cooling rate induced by the laser, in the range of $10^{7}-10^{8} \mathrm{~K} / \mathrm{s}$ [1,2], $\alpha^{\prime}$ martensitic phase forms in the Ti-6Al-4V. Elaboration process such as PB-LBM also induces localized high thermal gradient within the part $[3,4]$. Mismatches in dilatation caused by the thermal gradient generates RS [5] resulting in a stress state were the bulk is in a compressive state and last layers in tensile state [6,7]. Since this stress state can be a limit for part production in AM, RS determination is an important concern. Such determination is often performed by XRD technique using the $\sin ^{2} \psi$ method [8]. This technique allows to relate the variation of a given lattice plane spacing to local stress components. This requires the knowledge of the XEC $S_{1}$ and $1 /{ }_{2} S_{2}$ of the given lattice plane for the phase under consideration. The XEC are connected to Young modulus and Poisson ratio for the considered lattice plane through the relations:

$$
S_{1}^{\{h k l\}}=-\frac{v_{\{h k l\}}}{E_{\{h k l\}}} \text {, and } \frac{1}{2} S_{2}^{\{h k l\}}=\frac{1+v_{\{h k l\}}}{E_{\{h k l\}}} .
$$

The present study concerns the Ti-6Al-4V alloy which is widely used in industry and which is the most used titanium alloy for AM. After processing via LBM process the microstructure of the alloy is mainly constituted by the $\alpha^{\prime}$ martensitic phase, as shown in Fig. 1. This $\alpha^{\prime}$ martensitic 
phase presents the same hexagonal structure than the $\alpha$ phase but different composition since it incorporates $\beta$-forming elements such as vanadium. In order to measure the RS in LBM parts of Ti-6Al-4V alloys one needs to know the values of XEC for this specific $\alpha$ ' martensitic phase. However, to our knowledge the only XEC values available in the literature for Ti alloys refer to either pure Ti $\alpha$ phase [9] or one lattice plane of the $\alpha$ phase of a wrought Ti-6Al-4V alloy [10]. In this paper, a methodology is proposed and discussed to determine XEC values of the $\alpha$ ' martensitic phase, by performing synchrotron X-ray diffraction on tensile specimens elaborated by PB-LBM process. The values are compared to the literature data.

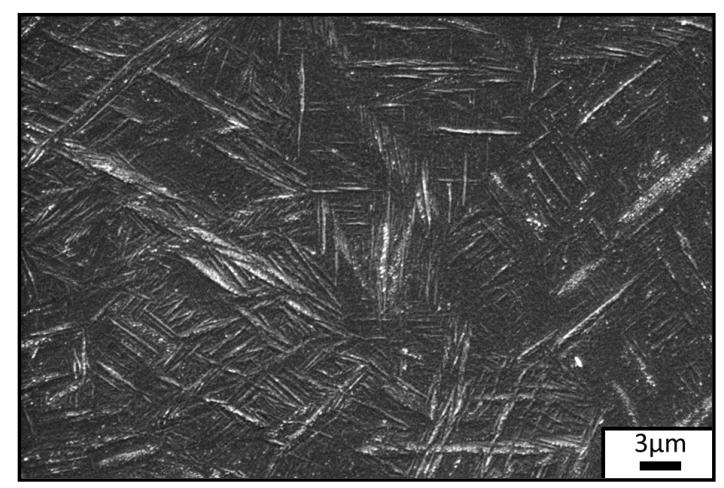

Figure 1 - $\alpha$ ' martensitic microstructure of the Ti-6Al-4V processed by LBM

\section{XEC Determination Method}

The method to determine the XEC is based on in-situ diffraction measurements during a tensile test within the elastic domain of the given specimen [8]. Three samples were directly fabricated at the desired shape and dimensions by LBM using M2 Cusing machine. The powder used was reused Ti-6Al-4V powder with an average diameter of $35 \mu \mathrm{m}$. Samples have been manufactured vertically using standard melting parameters provided by machine manufacturer (layer thickness: $60 \mu \mathrm{m}$ ). The sample thickness is $2 \mathrm{~mm}$, the gauge length is $2 \mathrm{~mm}$ wide and $10 \mathrm{~mm}$ in length. For a better diffraction signal all the sample surfaces were grinded using SiC grade 600 paper, as a result thickness and width of the gauge length decrease and were measured for each sample: 1.86 $\mathrm{x} 1.87 \mathrm{~mm}^{2}$ for sample $1,1.90 \times 1.86 \mathrm{~mm}^{2}$ for sample 2 and $1.84 \mathrm{x} 1.85 \mathrm{~mm}^{2}$ for sample 3 . The tensile tests were performed using a load frame electromechanical driven system that can be adapted to beamline ID11 at the ESRF. A maximal loading of $3 \mathrm{kN}$ is allowed with one mobile jaw. The load was changed step by step $(500 \mathrm{~N})$, first increased from 0 up to $2000 \mathrm{~N}$, then decreased back to $0 \mathrm{~N}$. Each step corresponds to a holding time of 25 seconds during which the diffraction rings are recorded. The maximum load (2000 N) corresponds to a tensile stress of 575 MPa for sample 1, $566 \mathrm{MPa}$ for sample 2 and $588 \mathrm{MPa}$ for sample 3, which is well below the yield stress of the alloy in the raw fabrication state estimated to be $1100 \mathrm{MPa}$. Diffraction data were recorded during both loading and unloading steps showing similar results, further calculations are obtained from the analysis of unloading data. These measurements were performed at the ESRF, on the beamline ID11 with a wavelength of $0.0140891 \mathrm{~nm}(\mathrm{E}=88 \mathrm{keV})$, a beam size of $300 \times 400 \mu^{2}$ and diffraction patterns were acquired on a 2D detector (FreLon camera). Such 2D diffraction pattern with indication of the $\delta$ and $\theta$ angles are represented in Fig. 2a. $\delta$ is the angle along the ring, $\delta=90^{\circ}$ corresponding to the tensile axe. The classical $\psi$ angle is simply deduced from $\delta$ using $\psi=\delta-\pi / 2$ [11]. The $\theta$ angle is the Bragg's Angle. Diffraction patterns were integrated from $0^{\circ}$ to $90^{\circ}$ every $10^{\circ}$ in $\psi$ angles, within a sectorial window of ${ }^{+} / 5^{\circ}$. The four quarters were added for a better signal to noise ratio and get an average value for each $\psi$ angle. The resulting diffraction patterns is presented on Fig.2b. 

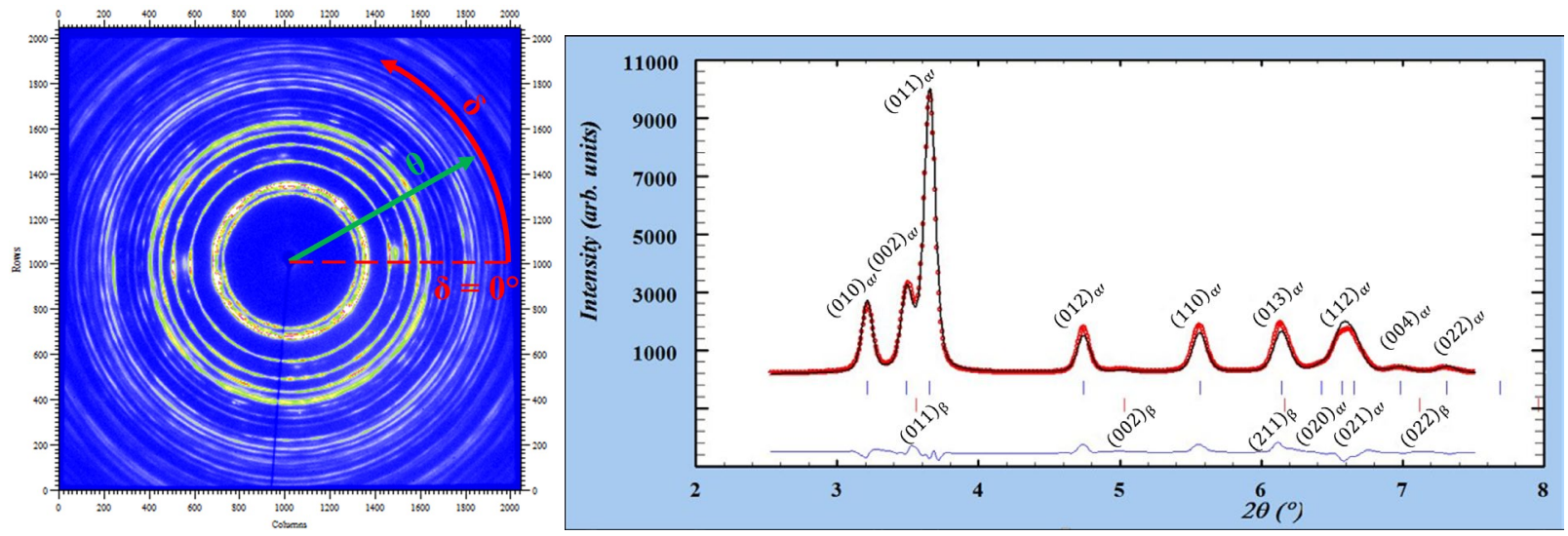

Figure 2 - Example of a diffraction pattern a) as recorded on the $2 D$ detector with the angles $\delta$ and $\theta$, and $b$ ) integrated over $\psi$ sector.

From these diffraction patterns the lattice spacing $d_{h k l}$ for different orientations were obtained using Bragg's law. The corresponding strain values were calculated using Eq. 2 were $\mathrm{d}_{0, \mathrm{hkl}}$ is the reference spacing taken from diffraction patterns at $0 \mathrm{~N}$ load.

$$
\varepsilon_{h k l} \approx \frac{d_{h k l}-d_{0, h k l}}{d_{0}, h k l} .
$$

This deformation varies with the angle $\psi$ according to Eq. 3, with $\sigma_{1 R}$ and $\sigma_{2 R}$ being the RS in the tensile and transverse directions respectively and $\sigma_{A}$ the applied stress [12].

$$
\varepsilon_{\psi, h k l}=\frac{1}{2} S_{2}^{\{h k l\}}\left(\sigma_{A}+\sigma_{1 R}\right) \sin ^{2} \psi+S_{1}^{\{h k l\}}\left(\sigma_{A}+\sigma_{1 R}+\sigma_{2 R}\right) .
$$

Because the RS state does not change during the loading and due to the high energy of the X-ray beam and the transmission geometry, the whole beam path was analyzed; residual stresses can be neglected as the low load state $(0 \mathrm{~N})$ is taken as a reference in order to determine the XEC, resulting in:

$$
\varepsilon_{\psi, h k l}=\frac{1}{2} S_{2}^{\{h k l\}} \sigma_{A} \sin ^{2} \psi+S_{1}^{\{h k l\}} \sigma_{A} .
$$

Note that in the sections below that concern results on hexagonal phase the lattice plane are designated using the four indices ( $h k i \ell$ ) - where the redundant index i is defined by $h+k+i=0$.

\section{Results}

The procedure used for the determination of XEC values is first described for the lattice plane (1013) on sample 1. The deformation is first plotted against $\sin ^{2} \psi$ for the different stress levels. According to Eq. 4, the deformation may obey a linear relation to the $\sin ^{2} \psi$, the fitting of the experimental points in Fig. 3 shows that this relation is roughly verified. In this plot, one can see that the linear fit corresponding to different values of stress level intersect each other on an invariant point. Indeed, if one sets a zero deformation, Eq. 4 can be simplified and gives:

$$
\varepsilon=0=\frac{1}{2} S_{2} \sigma_{A} \sin ^{2} \psi+S_{1} \sigma_{A}=>\frac{1}{2} S_{2} \sin ^{2} \psi=-S_{1}
$$

This unique intersection point for several fitting lines is taken as an indication of coherent experimental results. Similar results were obtained, as for Figs. 3 and 4, for all the (hkil) lattice planes and specimens analyzed. Using Fig. 3 and Eq. 4, XEC can be accessed by plotting the slope of fitting lines (Fig. 4a) and their intercept (Fig. 4b) versus the applied stress. The resulting data in the graph of Fig. $4 \mathrm{a}$ and $\mathrm{b}$ shows a clear linear dependence against the applied stress. According to Eq. 4 the slope of these lines corresponds respectively to $S_{1}$ and $1 / 2 S_{2}$. 


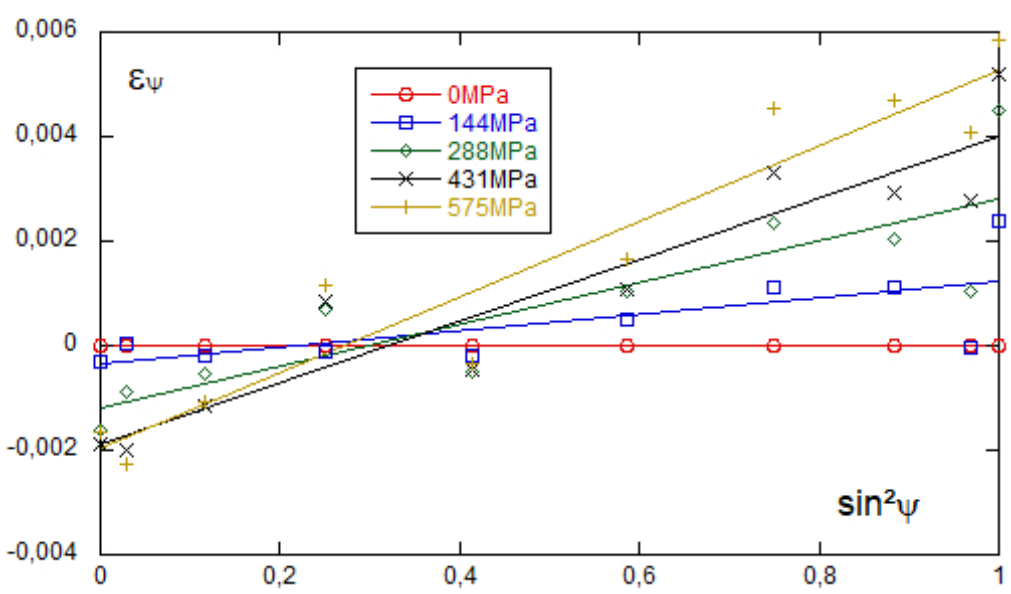

Figure 3 - Deformation $\varepsilon_{\psi}$ versus $\sin ^{2} \psi$ for (1013) for different stress values in sample 1
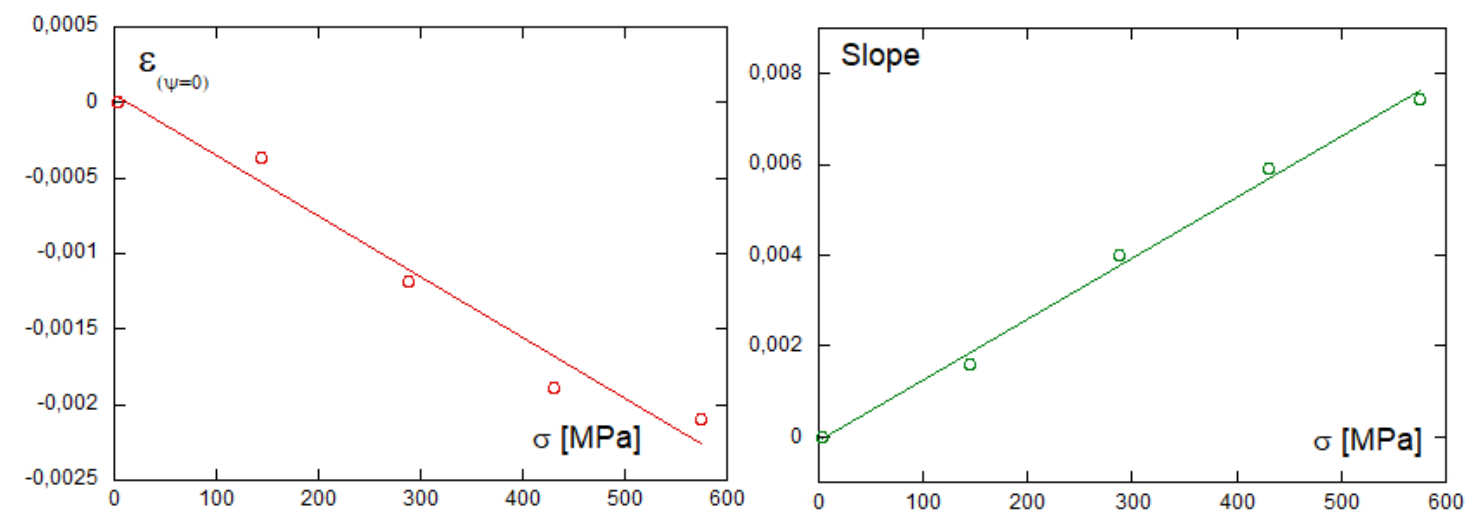

Figure 4-Line fitting data from Fig. 3: a) Intercept and b) slope versus the applied stress.

Table 1 -XEC in $10^{-6}(\mathrm{MPa})^{-1}$ experimentally determined for the different specimens.

\begin{tabular}{|c|c|c|c|c|c|c|c|c|c|c|}
\hline & \multicolumn{2}{|c|}{$(11 \overline{2} 0)$} & \multicolumn{2}{|c|}{$(10 \overline{1} 1)$} & \multicolumn{2}{|c|}{$(10 \overline{1} 2)$} & \multicolumn{2}{|c|}{$(10 \overline{1} 3)$} & \multicolumn{2}{|c|}{$(0002)$} \\
\hline Sample & $\mathrm{S}_{1}$ & $1 /{ }_{2} S_{2}$ & $\mathrm{~S}_{1}$ & $1 /{ }_{2} S_{2}$ & $\mathrm{~S}_{1}$ & $1 /{ }_{2} S_{2}$ & $\mathrm{~S}_{1}$ & $1 /{ }_{2} \mathrm{~S}_{2}$ & $\mathrm{~S}_{1}$ & $1 / 2 S_{2}$ \\
\hline 1 & -5.00 & 16.30 & -4.69 & 15.40 & -4.26 & 14.10 & -4.00 & 13.40 & -3.64 & 12.30 \\
\hline 2 & -3.82 & 11.70 & -3.71 & 11.40 & -3.55 & 11.00 & -3.45 & 10.80 & -3.32 & 10.50 \\
\hline 3 & -2.98 & 12.40 & -2.55 & 11.70 & -1.94 & 10.60 & -1.58 & 10.00 & -1.05 & 9.14 \\
\hline
\end{tabular}

The same procedure was applied for five different (hkil) planes in the three studied samples, giving rise to the whole set of XEC results showed in Table 1 . The values presented in Table 1 shows that XEC are quite different from one specimen to the other. A possible reason for this scattering could be the orientation texture of the samples. Textured materials can no longer be described accurately by XEC, but described by X-Ray Elastic Factors (XEF) $\mathrm{F}_{\mathrm{ij}}$ [8]. A way to get an idea of the texture is to plot the XEF component $F_{11}$, which is the slope of the applied stress against the deformation $\varepsilon_{\psi}$ for each value of $\sin ^{2} \psi$. Such a plot is showed in Fig. 5 for the plane (1013) in sample 1. In such a plot, a non-textured material would result in a linear fit of $F_{11}$ as indicated by the dashed line in Fig. 5, whereas the oscillations observed in this plot (full line) may reveals the existence of orientation texture. However, the limited number of stress levels (5 steps) used to draw this plot may contribute to scattering seen in Fig. 5. Other possible origin for the variation of XEC on different samples may arise from a more general variation in 
microstructure or different porosity level due to different position of the specimens on the elaboration plates.

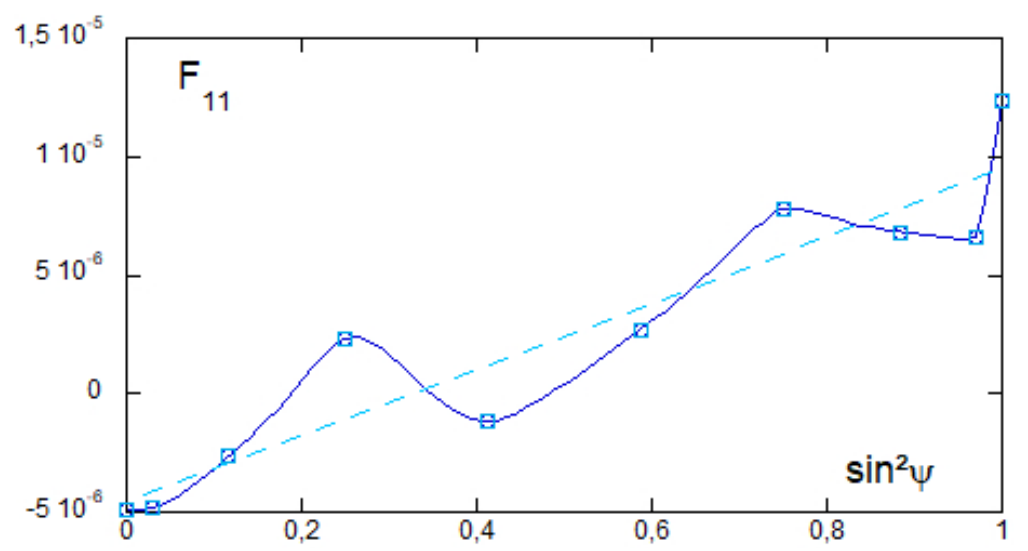

Figure 5 - X-Ray Elastic Factors $F_{11}$ for the loading of sample 1

Nevertheless, in order to pursue the analysis of the experimentally determined XEC values, the mean values of $S_{1}$ and $1 / 2 S_{2}$ were calculated for each lattice plane. Those values are shown in Table 2 together with the XEC values available in the literature for the $\alpha$ phase of pure titanium [9] and $\alpha$ phase from wrought Ti-6Al-4V [10].

Table 2 - XEC experimentally determined and from literature in $10^{-6}(\mathrm{MPa})^{-1}$

\begin{tabular}{|c|cc|cc|cc|cc|cc|}
\hline & \multicolumn{2}{|c|}{$(11 \overline{2} 0)$} & \multicolumn{2}{c|}{$(10 \overline{1} 1)$} & \multicolumn{2}{c|}{$(10 \overline{1} 2))$} & \multicolumn{2}{c|}{$(10 \overline{1} 3)$} & \multicolumn{2}{c|}{$(0002)$} \\
\hline & $\mathrm{S}_{1}$ & $1 /{ }_{2} \mathrm{~S}_{2}$ & $\mathrm{~S}_{1}$ & $1 /{ }_{2} \mathrm{~S}_{2}$ & $\mathrm{~S}_{1}$ & $1 /{ }_{2} \mathrm{~S}_{2}$ & $\mathrm{~S}_{1}$ & $1 /{ }_{2} \mathrm{~S}_{2}$ & $\mathrm{~S}_{1}$ & $1 /{ }_{2} \mathrm{~S}_{2}$ \\
\hline$\alpha$ ' This study & $-\mathbf{3 . 9 3}$ & $\mathbf{1 3 . 4 7}$ & $\mathbf{- 3 . 6 5}$ & $\mathbf{1 2 . 8 3}$ & $\mathbf{- 3 . 2 5}$ & $\mathbf{1 1 . 9 0}$ & $\mathbf{- 3 . 0 1}$ & $\mathbf{1 1 . 4 0}$ & $-\mathbf{2 . 6 7}$ & $\mathbf{1 0 . 6 5}$ \\
$\alpha$ pure Ti [9] & -2.98 & 12.03 & -2.90 & 11.80 & -2.74 & 11.28 & -2.56 & 10.83 & -2.34 & 10.16 \\
$\alpha$ W Ti64 [10] & & & -3.26 & 13.38 & & & & & & \\
\hline
\end{tabular}

First, a clear variation of the XEC values with the lattice plane can be noticed, evidencing the elastic anisotropy of the hexagonal structure, with (absolute) values of XEC decreasing when changing from prismatic to basal plane. This tendency is visible for together the $\alpha$ ' martensitic phase (this study) and the pure Ti $\alpha$ phase, however the anisotropy seems to be stronger for the previous one. Secondly, large differences between $\alpha$ pure titanium phase and $\alpha$ ' martensitic phase of the Ti-6Al-4V in means of XEC are observed. The difference between both sets of values is stronger close to the prismatic lattice plane and can reach $32 \%$ for $S_{1}^{11 \overline{2} 0}$ and $12 \%$ for $\frac{1}{2} S_{2}^{11 \overline{2} 0}$. This difference reduces to $14 \%$ for $S_{1}^{0002}$ and $5 \%$ for $\frac{1}{2} S_{2}^{0002}$ for basal plane. This difference in XEC values seems also reduced when the present results are compared to the only value that could be found for the $\alpha$ phase from wrought Ti-6Al-4V (W Ti64) for the (1011) plane. Work is in progress to get a better understanding about the variation of elastic properties of the $\alpha$ hexagonal $\mathrm{Ti}$ phase depending on processing method and chemical composition [13].

\section{Conclusion}

In this study, the XEC were experimentally determined from the particular $\alpha$ martensitic phase of the Ti-6Al-4V elaborated through AM process, using a methodology based on XRD and 2D detector. Results obtained from the three different specimens shown a quite large scattering that could be related to microstructural variation of specimens. Measurement of XEC for 5 lattice planes evidenced the orientation variation of $S_{1}$ and $1 / 2 S_{2}$ from prismatic planes to basal planes, in good agreement with the anisotropy of the $\alpha$ phase seen in previous study. Results shown that there are differences between XEC determined for the $\alpha^{\prime}$ martensitic phase of the Ti-6Al-4V and those of the $\alpha$ pure titanium phase usually used for RS determination. This may lead to errors in RS values determined by $\sin ^{2} \psi$ method that could be higher than $10 \%$ for the $(11 \overline{2} 0)$ plane. 


\section{Acknowledgement}

The author would like to acknowledge M. Dehmas and M. François for their assistance and fruitful discussions.

\section{References}

[1] B. Vrancken, V. Cain, R. Knutsen, J. Van Humbeeck, Residual stress via the contour method in compact tension specimens produced via selective laser melting, Scripta Mater. 87 (2014) 29-32. https://doi.org/10.1016/j.scriptamat.2014.05.016

[2] J. Yang, H. Yu, J. Yin, M. Gao, Z. Wang, X. Zeng, Formation and control of martensite in Ti-6Al-4V alloy produced by selective laser melting, Materials and Design 108 (2016) 308-318. https://doi.org/10.1016/j.matdes.2016.06.117

[3] A. Vasinonta, J.L. Beuth, M. Griffith, Process maps for predicting residual stress and melt pool size in the laser-based fabrication of thin-walled structures, J. Manuf. Sci. Eng 129 (2006) 101-109. https://doi.org/10.1115/1.2335852

[4] G. Vastola, G. Zhang, Q.X. Pei, Y.-W. Zhang, Controlling of residual stress in additive manufacturing of Ti6Al4V by finite element modeling, Add. Manufacturing 12 (2016) 231-239. https://doi.org/10.1016/j.addma.2016.05.010

[5] Y. Liu, Y. Yang, D. Wang, A study on the residual stress during selective laser melting of metallic powder, Int. J. Adv. Manuf. Tech. 87 (2016) 647-656. https://doi.org/10.1007/s00170016-8466-y

[6] J. Grum, R. Sturm, A new experimental technique for measuring strain and residual stresses during a laser remelting process, J. of Mat. Processing Tech. 147 (2016) 351-358. https://doi.org/10.1016/j.jmatprotec.2004.01.007

[7] P. Mercelis, J.-P. Kruth, Residual stresses in selective laser sintering and selective laser melting, Prototyping Journal, 12 (2006) 254-265. https://doi.org/10.1108/13552540610707013

[8] V. Hauk, Structural and Residual stress analysis by nondestructive methods, Elsevier, Amsterdam, 1997, Chapter 13

[9] B. Eigenmann, E. Macherauch, Rontgengraphische Untersuchung von Spannungszustanden in Werkstoffen, Teil III, Material-wissenschaft und werkstofftechnik, Bd.27, (1995) 426-437. https://doi.org/10.1002/mawe.19960270907

[10] G. Bruno, B. Dunn, The precise measurement of Ti6Al4V microscopic elastic constants by means of neutron diffraction, Meas. Schi. Technol. 8 (1997) 1244-1249. https://doi.org/10.1088/0957-0233/8/11/006

[11] L. Vautrot, G. Geandier, M. Mourot, M. Dehmas, E. Aeby-Gautier, B. Denand, S. Denis, Internal stresses in Metal Matrix Composites in relation with matrix phase transformations, Advanced Materials Research, 996 (2014) 944-950. https://doi.org/10.4028/www.scientific.net/AMR.996.944

[12] V. Ji, Contribution à l'analyse par diffraction des rayons X de l'état microstructural et mécanique des matériaux hétérogène, Habilitation à Diriger des Recherche, Université des Sciences et Technologies de Lille (2003)

[13] N. Dumontet, D. Connetable, B. Malard, B. Viguier, Elastic properties of the $\alpha$ ' martensitic phase of the Ti-6Al-4V, to be published 